\title{
Nanomutilla vaucheri in Sardegna, prima segnalazione per l'Italia (Hymenoptera Mutillidae)
}

\begin{abstract}
Riassunto - Viene resa nota la presenza in Sardegna di Nanomutilla vaucheri (Tournier, 1895), in base alla raccolta di un esemplare di sesso femminile. Questo reperto costituisce la prima segnalazione per il territorio italiano di un rappresentante della sottofamiglia Ticoplinae. La specie è segnalata per la prima volta anche per la Tunisia. Vengono riportate alcune osservazioni sulla biologia di questa specie e ne viene fornita una breve ridescrizione. Infine sono aggiunte alcune note tassonomiche e sistematiche sul genere Nanomutilla André, 1900.
\end{abstract}

\begin{abstract}
Nanomutilla vaucheri in Sardinia, first record from Italy (Hymenoptera Mutillidae).
A single female of Nanomutilla vaucheri (Tournier, 1895) is reported from Sardinia, being the first record of a representative of the subfamily Ticoplinae from Italy. The species is recorded for the first time from Tunisia, too. Observations on its biology, together with a short redescription of the species, are provided. The paper is concluded by taxonomic notes on the genus Nanomutilla André, 1900.
\end{abstract}

Key words: Mutillidae, Ticoplinae, Nanomutilla, Sardinia.

\section{INTRODUZIONE}

Fra i tanti interessanti e talora inediti materiali che la Sardegna, anche fra gli Imenotteri Mutillidae, ancora custodisce, ho recentemente ricevuto, grazie alla disponibilità dell'amico Piero Leo, noto entomologo residente a Cagliari, un piccolissimo esemplare $(2,3 \mathrm{~mm})$ di sesso femminile raccolto in Sardegna dal compianto Carlo Meloni, che non corrisponde a nessuna delle specie di Mutillidae ad oggi note per l'Italia.

L'attento esame dei caratteri morfologici e, soprattutto, il confronto col materiale tipico di Marocco presente al Museo di Ginevra e con un esemplare di Tunisia conservato presso il Museo civico di Storia naturale "G. Doria" di Genova, mi hanno permesso di identificarlo come Nanomutilla vaucheri (Tournier, 1895) (Mutillidae Ticoplinae).

Questo reperto costituisce la prima segnalazione per il territorio italiano di un rappresentante di questa sottofamiglia di Mutillidae e lascia presupporre una sua possibile presenza in altre regioni italiane.

Anche la Tunisia va aggiunta all'areale sino ad ora noto per questa specie, sulla base di un unico esemplare, già così determinato da F. Invrea, che ho avuto modo di esaminare personalmente, presente in coll. Gribodo, presso il Museo civico di Storia naturale "G. Doria” di Genova.

L'apparente rarità di $N$. vaucheri è certamente attribuibile alle sue ridotte dimensioni ed è in parte spiegabile, sulla base delle poche osservazioni in campo riportate in letteratura, sulla scorta del particolare ambiente in cui la specie si raccoglie.

In particolare Giner Marì (1944), nel citarla per la Spagna, scrive: "Esta especie, del norte de Africa y del Asia Menor, se encuentra también en España, donde puede que esté bastante difundida por cierta parte del litoral mediterráneo. La extremada pequeñez de sus individuos dificulta su hallazgo y hace que pase inadvertida en muchos lugares en donde puede hallarse. Por otra parte, como se refugia entre la hojarasca y las raicillas de algunas plantas, su captura es difícil y exige una atención particular a que no es frecuente se entregue en el campo el cazador de himenópteros. Buscando coleópteros pequeñisimos, la ha encontrado en Tánger el señor Escalera, refugiada bajo las hojas caulinares de un pequeño Cystus (sic!) de flor blanca, que allí es bastante abundante. En Gibraltar se han encontrado por varios coleopterólogos buscadores de pequeños Clavicornios".

Non sono riportati, fra i dati che accompagnano l'esemplare sardo, quelli riferiti alle modalità del ritrovamento.

Era nota comunque l'attenzione che il compianto Carlo Meloni dedicava, fra l'altro, alla raccolta mediante vaglio dei microcoleotteri e dei microimenotteri atteri presenti nel terreno e di cui sono testimonianza le lunghe serie presenti nella sua raccolta, oggi conservata presso il Museo civico di Storia naturale "G. Doria" di Genova e che ho avuto il piacere di esaminare recentemente. 
Ritengo sia utile, data la frammentarietà e la scarsità delle informazioni che la riguardano, fornire una breve ridescrizione di questa specie, di cui non è ancora noto il sesso maschile, e riassumere i dati a tutt'oggi noti relativi al genere Nanomutilla André, 1900.

\section{Note Morfologiche}

Esemplari esaminati. Olotipo $\bigcirc$ : Tangeri, Vaucher leg. (Muséum d'Histoire naturelle, Genève); 1 ๆ Tangeri, 10.1896, L. Berland det. 1922 (Muséum d'Histoire naturelle, Genève); 1 \& Tunisi, Coll. Gribodo, Nanomutilla vaucheri Tour. - Determ. F. Invrea (Museo civico di Storia naturale "G. Doria" di Genova); 1 ㅇ Libia, Tripolitania, El Gusbat, 7.5.1936, R. e C. Koch (Museo civico di Storia naturale di Milano); 1 Cabras (OR), Is Arutas, 30.III.2001, C. Meloni leg. (coll. M. Romano) (fig. 1)

Dimensioni: 2,3-2,5 $\mathrm{mm}$. Le dimensioni dell'esemplare tipico sono di $2,3 \mathrm{~mm}$ e non di 1,5 $\mathrm{mm}$, come indicato nella descrizione originale da Tournier (1895).

Tegumento nero. Zampe, antenne, mandibole, bordo posteriore dei tergiti e ultimo segmento addominale rosso ruggine. Femori brunastri. Capo e mesosoma rivestiti superiormente da una pubescenza coricata giallastra mediocremente densa e fine e con alcune lunghe setole erette dello stesso colore. Nel mesosoma la pubescenza coricata converge al centro verso la carena mediana. Metasoma coperto di una pubescenza fine, grigia, molto lunga, ma poco fitta, un po' sollevata; questa pubescenza forma, condensandosi al bordo posteriore dei segmenti, una fascia chiara stretta e poco nettamente definita. Punteggiatura fine e densa su tutta la superficie di capo, mesonoto e metanoto, conferendole un aspetto pressoché opaco.

Capo, in visione dorsale, subsferico, poco più largo del mesosoma, fortemente prolungato dietro gli occhi, un po' compresso in visione laterale. Gli occhi, formati da ommatidi relativamente grandi e poco numerosi, sono molto più vicini all'articolazione delle mandibole che agli angoli posteriori del capo. Antenne robuste, inserite molto prossime l'una all'altra e direttamente sotto il clipeo, primo articolo del funicolo leggermente più lungo dei successivi, che si presentano, tranne l'ultimo, tanto larghi quanto lunghi.

Mesosoma allungato (rapporto lunghezza/larghezza: 1,35-1,40), a lati subparalleli, leggermente divergenti all'indietro, col bordo anteriore arcuato. Esso è interamente percorso al centro da una sottile carena longitudinale, visibile solo a forte ingrandimento e terminante posteriormente in un minuscolo ma evidente denticolo. Non vi è traccia di unguicolo scutellare.

Tibie esternamente inermi.

Propodeo nettamente troncato posteriormente, col bordo molto evidente, munito, a ciascun lato, di un piccolo dente acuminato.

Metasoma sessile, ovale allungato, col primo segmento troncato in avanti, a lati paralleli, largo all'estremità posteriore quanto il secondo, che è di

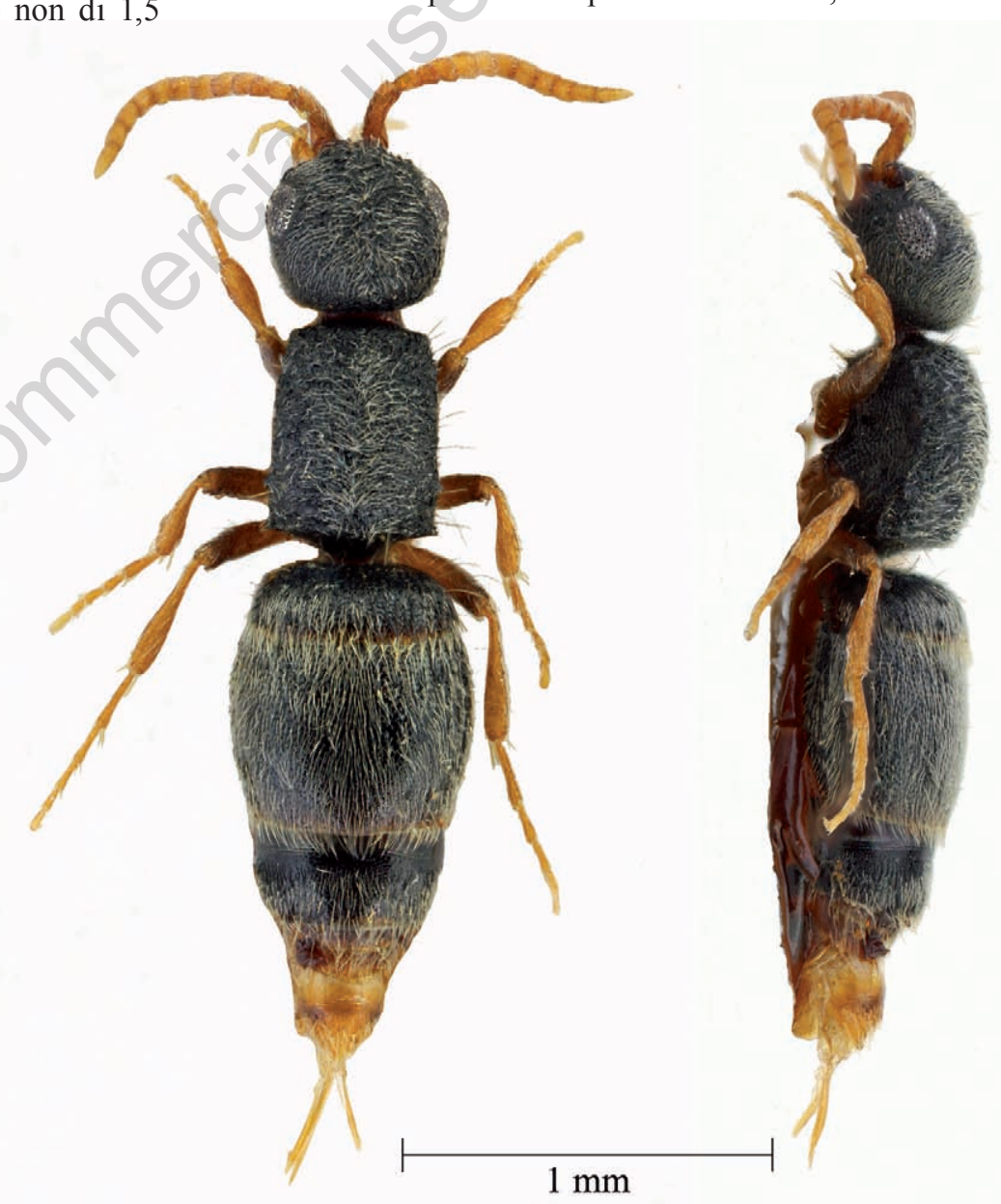

Figura 1. Nanomutilla vaucheri (Tournier) ( $९$; Oristano: Cabras): habitus (visione dorsale e laterale). 
forma allungata e presenta i lati debolmente convessi. Ultimo segmento lucido, senza area pigidiale.

La distribuzione nota della specie, prima dell'attuale contributo, che ne estende l'areale alla Tunisia e alla Sardegna, comprendeva Marocco (Tangeri, località tipica), Spagna meridionale (Gibilterra) e Algeria (André 1899-1903); Siria (André, 1902); Libia (Menozzi, 1940). Suárez (1975) ritiene però che le segnalazioni per la Siria vadano riferite ad altra specie.

Note Sistematiche e Tassonomiche sul Genere NaNOMUTILLA

Mutilla vaucheri viene descritta da Tournier (1895) sulla base di un esemplare di sesso femminile raccolto a Tangeri (Marocco) da M. H. Vaucher, cui la dedica. L'Autore sottolinea come questa specie dovrà, una volta noto anche il maschio, costituire un gruppo distinto nel genere Mutilla.

André (1899-1903) istituisce per questa specie il nuovo sottogenere Nanomutilla e segnala $M$. (N.) vaucheri anche di Spagna meridionale (Gibilterra) e Algeria (Mont Edough).

Successivamente lo stesso André (1902) eleva Nanomutilla al rango generico.

Nanomutilla è uno dei sei generi che compongono la sottofamiglia Ticoplinae Nagy, 1970, che riunisce al suo interno un gruppo limitato di specie, comprendente i taxa più primitivi fra i Mutillidae (Brothers, 1975; Lelej \& Nemkov, 1997).

Fra le caratteristiche considerate primordiali, proprie di questo gruppo, va ricordata la presenza di corte setole sugli occhi, in entrambi i sessi.

Al genere Nanonutilla sono attribuite, allo stato attuale, nella regione paleartica occidentale, quattro specie (Lelej, 2002). Due di esse sono ad oggi note nel solo sesso femminile: Nanomutilla vaucheri e Nanomutilla nadae Argaman, 1988, descritta di Spagna. Nanomutilla parila (Nagy, 1970) e Nanomutilla yoca (Nagy, 1970) sono le altre due specie note all'interno del genere, descritte entrambe di Giordania su esemplari di sesso maschile. Successivamente
Argaman (1988) descrive anche le presunte femmine di queste due specie, senza tuttavia riportare alcuna prova o le ragioni che lo hanno portato a riconoscere tale associazione maschio/femmina. L'identità dei tre taxa descritti da Nagy (1970) e da Argaman (1988) resta comunque assai difficile da chiarire, in quanto le descrizioni originali sono assai lacunose e i materiali tipici sono purtroppo andati perduti ${ }^{1}$.

Va infine aggiunto che, come scrivono Mitchell \& Brothers (2002), regna ancora molta confusione sulla reale identità di diversi esemplari identificati dagli Autori come Nanomutilla vaucheri. In particolare uno dei due Autori (D. J. Brothers), che per primo ha avuto la possibilità di esaminare il materiale tipico di Nanomutilla vaucheri, conservato presso il Muséum d'Histoire naturelle di Ginevra, evidenzia come Nonveiller (1973), Suarez (1975) e Argaman (1988) abbiano mal interpretato i caratteri propri di questa specie e che dunque, gli esemplari cui fanno riferimento nei propri contributi, e da loro indicati come Nanomutilla vaucheri, possano appartenere ad uno o più taxa diversi, ancora da descrivere.

Il genere necessita dunque di una revisione approfondita, resa indubbiamente assai problematica dalla difficile individuazione, in relazione alle piccolissime dimensioni dei suoi rappresentanti, di caratteri discriminanti ben visibili e costanti e dall'impossibilità di reperire i materiali tipici.

\section{Materiale Tipico}

Tournier (1895) nella sua descrizione originale sembra riferirsi ad un unico esemplare di sesso femminile raccolto da M. H. Vaucher a Tangeri (Marocco).

Lelej (2002), nel suo catalogo dei Mutillidi Paleartici, scrive, a proposito di questa specie: "syntypes probably in Museum d'Histoire Naturelle Genève".

Nello stesso anno Brothers (in Mitchell \& Brothers, 2002) riferisce di avere trovato ed esaminato due soli esemplari in collezione Tournier, presso il Museo di Storia Naturale di Ginevra e parla di uno dei due, quello incollato sul dorso, come dell'olotipo,

\footnotetext{
1) Credo sia utile qui chiarire che i due Autori Nagy e Argaman, citati sempre separatamente nelle bibliografie, sono in realtà la stessa persona.

- Carol Nagy operava presso la "Marine Research Station" di Agigea (Romania) e pubblicò a cavallo fra gli anni 1960 e 1970 . Nel 1981 dovette trasferirsi con la famiglia in Israele e cambiò il proprio cognome prima in Grosman e poi, definitivamente, in Argaman. Qabir (Carol) Argaman divenne in seguito curatore della collezione entomologica dell’Università di Tel Aviv a Bet Dagan e riprese a pubblicare negli anni '80 sotto questo nuovo cognome. Purtroppo, alla sua morte, come mi è stato riferito da Laibale Friedman, curatore delle collezioni del Museo dell'Università di Tel Aviv, la collezione è andata smembrata e in parte venduta a privati dagli eredi, in parte lasciata presso il Ministero dell'Agricoltura, dove Argaman lavorava. Tuttavia, come mi scriveva lo stesso Dr. Friedman "for some unknown reason Argaman removed labels from part of the specimens including types, leaving them without any label". Questo ha reso perciò impossibile ritrovare, malgrado le accurate ricerche, il materiale tipico relativo alle specie del genere Nanomutilla descritte dallo stesso Argaman.
} 
per la presenza di un cartellino autografo di Tournier con la determinazione e il riferimento alla sua stessa pubblicazione (fig. 2). Il secondo esemplare risulta raccolto nel 1896, dopo la pubblicazione della descrizione originale e dunque non può essere un sintipo, anche se topotipico.

Avendo anch'io accertato che non sono presenti altri esemplari in collezione Tournier concordo con le conclusioni di Brothers di considerare l'esemplare incollato sul dorso come l'olotipo di Nanomutilla vaucheri Tournier, 1895.

\section{RINGRAZIAMENTI}

Un sincero ringraziamento a Piero Leo di Cagliari per la fiducia accordatami, a Roberto Poggi, Direttore del Museo civico di Storia naturale di Genova, per l'attenta lettura del manoscritto e i preziosi consigli, a Maurizio Pavesi, del Museo civico di Storia naturale di Milano, a Bernhard Merz, conservatore presso il Muséum d'Histoire naturelle di Ginevra, che mi ha messo a disposizione il materiale di quella Istituzione e a Laibale Friedman, curatore delle collezioni del National Museum of natural History, Department of Zoology, Tel Aviv University, per le utili informazioni trasmessemi.

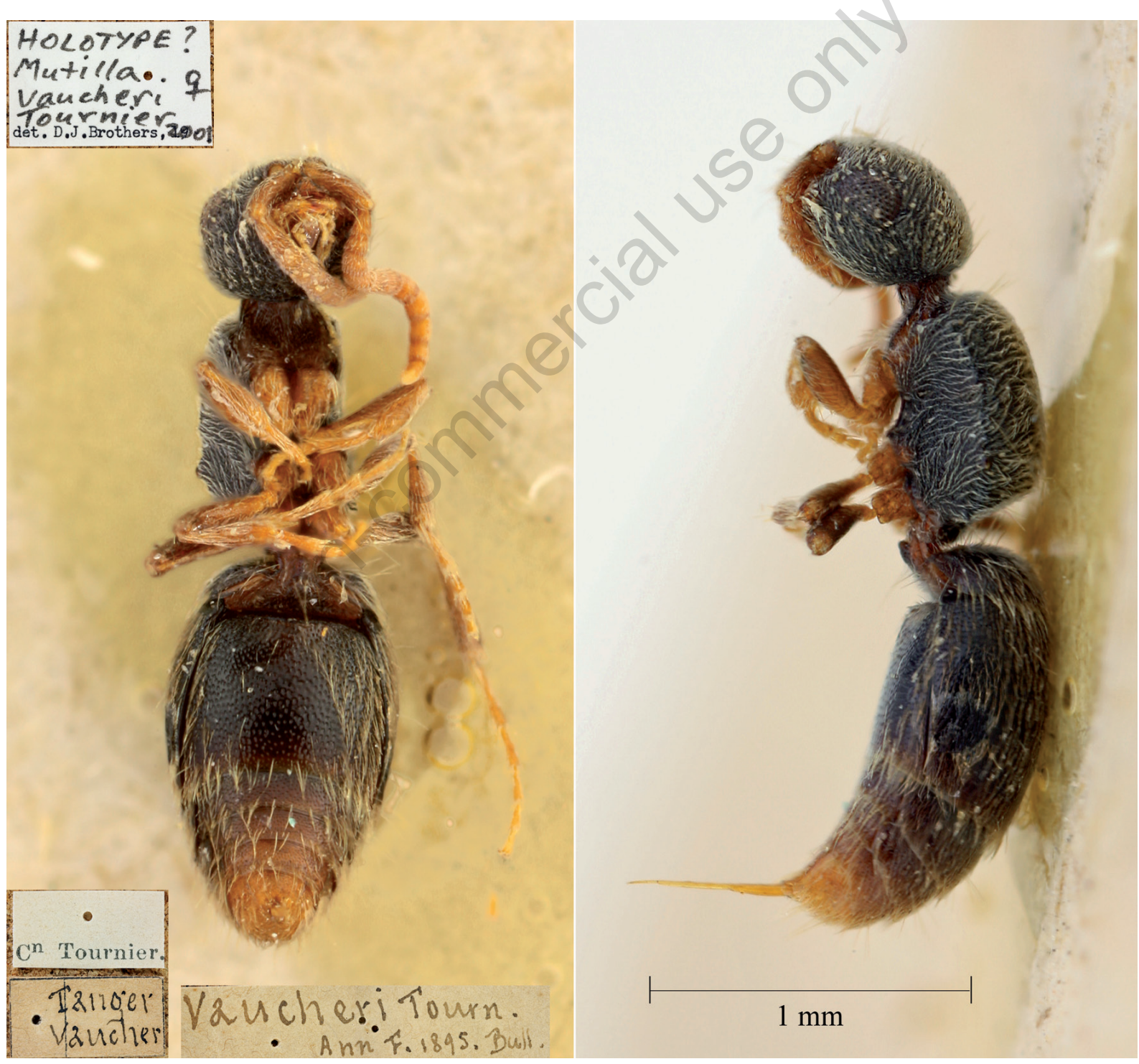

Figura 2. Nanomutilla vaucheri (Tournier), holotypus $ㅇ$ : habitus (visione dorsale e laterale). 


\section{BiBLIOGRAFIA}

André E., 1899-1903 - Species des Hyménoptères d'Europe \& d'Algérie. Les Mutillides. A. Hermann Ed., Paris, 8: 1899: 1-64, 1900: 65-144, 1901: 145-304, 1902: 305-384, 1903: 385-479 + pls. 1-15.

André E., 1902 - Fam. Mutillidae. In Wytsman, P.: Genera insectorum, Bruxelles, 11: 1-77, 3 pls.

Argaman Q., 1988 - Description of the female of Ticopla, with biological and taxonomic notes (Hymenoptera, Mutillidae). Fragmenta balcanica Musei macedonici scientiarum naturalium, 14(5): 33-45, 20 figs.

Brothers D. J., 1975 - Phylogeny and classification of the aculeate Hymenoptera, with special reference to Mutillidae. The University of Kansas Science Bulletin, 50(11): 483-648, 101 figs.

Giner Marí J., 1944 - Hymenópteros de España. Fams. Apterogynidae y Mutillidae. Trabajos del Instituto español de Entomologia, Madrid, 124 pp., 37 figs.

Lelej A. S. \& Nemkov P. G., 1997 - Phylogeny, evolution and classification of Mutillidae (Hymenoptera). Far Eastern Entomologist, 46: 1-24.

Lelej A. S., 2002 - Catalogue of the Mutillidae (Hymenoptera) of the Palaearctic region. Russian Academy of Sciences far Easter Branch Institute of Biology and Soil Science, Vladivostok: Dalnauka. 171pp.

Menozzi C., 1940 - Contributo alla fauna della Tripolitania. Bollettino del R. Laboratorio di Entomologia agraria di Portici, 31: $243-273$.

Mitchell A. \& Brothers D. J., 2002 - Phylogeny of the genera of Ticoplinae (Hymenoptera: Mutillidae). Journal of Hymenoptera Research, 11(2): 312-325

NAGY C. G., 1970 - Further investigations on the heterogynoid wasps. Entomologische Mitteilungen aus dem Zoologischen Museum Hamburg, 4(69): 83-86, 1 fig.

Nonveiller G., 1973 - Recherches sur les mutillides de l'Afrique (Hymenoptera, Mutillidae). III. Remarques concernant le genre Nanomutilla André 1899. Annales de la Faculté des Sciences du Cameroun, 15-16: 63-73, 3 figs.

SuÁrez F. J., 1975 - Comentarios sobre Smicromyrmilla Suarez y Nanomutilla André (Hymenoptera; Mutillidae). Archivos del Instituto de Aclimatación de Almería, 20: 105-119.

Tournier H., 1895 - Sur Rhinospathus Chobauti Desbr. (Coléoptères) et sur deux Mutilles nouvelles du Maroc (Hyménoptères). Bulletin de la Société entomologique de France, pp. XLVII-XLVIII.

Indirizzo dell'Autore:

M. Romano, Piazza A. Cataldo 10, I-90040 Capaci PA, Italia. marcellr@libero.it 